\title{
DENTAL MEDICINE AND PSYCHIATRY: THE NEED FOR COLLABORATION AND BRIDGING THE PROFESSIONAL GAP
}

\author{
Zdenko Šarac ${ }^{1}$, Ružica Zovko ${ }^{1}$, Marina Ćurlin² \& Pavo Filaković ${ }^{3}$ \\ ${ }^{1}$ Faculty of Dental Medicine, Department of Head and Neck Diseases, University of Mostar, \\ Mostar, Bosnia and Herzegovina \\ ${ }^{2}$ Faculty of Medicine, Department of Biochemistry, University of Mostar, Mostar, Bosnia and Herzegovina \\ ${ }^{3}$ Faculty of Dental Medicine and Health, University "Josip Juraj Strossmayer", Osijek, Croatia
}

received: 17.6.2019;

revised: 18.9.2019;

accepted: 20.2 .2020

\section{SUMMARY}

Dental health and mental health are strongly associated. Neglecting either of them can negatively influence on the other and induce many health and communication problems. Association between oral/dental health, self-esteem, quality of life and holistic health has been recognized for a long time. There has been increasing interest in dental health among patients with major mental disorders as well as in mental states and problems among patients with orodental disorders. Despite of huge progress in the field of dentistry psychiatric patients have had poor oral/dental health. Patients with major mental disorders have quite number of the risk factors for oral disease and consequently poorer dental health, but oral/dental problems and diseases are commonly overlooked or neglected. Bad or inappropriate dental care is related to the patients' amotivation, ignorance, fears, low economic status, stigmas and negative attitudes by the medical professionals. It is important to stress that dental diseases in psychiatric patients deserve the same attention as other comorbid somatic diseases. In this review we accentuate the need for more collaboration in order to bridge the professional gap between dentistry and psychiatry.

Key words: dental medicine - mental disorders - dental health - comorbidity - psychiatrist-dentist liaison service

$$
* * * * *
$$

\section{INTRODUCTION}

No mental health without oral health (Kisely 2016), as well as no oral health without mental health

Oral/dental health and mental health does not exist separately, and they affect each other in many ways. Comorbidity of mental disorders and somatic diseases has been in the focus of growing attention during the last several decades (Simunovic Filipcic \& Filipcic 2018, Jakovljevic \& Borovecki 2019). However, significantly less interest has been devoted to the oral health in patients with psychiatric disorders although oral/dental health is an important part of health in general and is closely associated with mental health. Poor dental/oral health has been related to somatic diseases such as diabetes, coronary heart disease and respiratory diseases, which may mediate negative effects on mental health (Arigbede et al. 2012). That's why comorbidity of mental disorders and dental diseases has become an important issue in the both, psychiatry and dental medicine. The lack of oral health evaluations and care in psychiatric patients requires more attention in clinical practice. It is well known fact that psychiatric patients are particularly exposed to high risk of dental problems and diseases associated with both patient-related and servicerelated factors due to specific characteristics of their mental disorder, comorbid somatic disease, life-style, cultural practice, stigma and social status (Grinshpoon et al. 2015). The knowledge and skill of the dentists in managing patients with mental disorders has been recognized as a professional barrier to oral health (Horst
1992). Good news is that many of these risk factors are modifiable. On the other side of the mental-dental health story, psychopathological states in dental patients are also usually unrecognized and hence untreated or not well enough treated. Good news is that there is a growing interest for psychiatric-dental liaison to address this unmet need as well as that the many of these patients may respond well to simple and effective interventions (see also Bathia et al. 2015).

The aim of this narrative review is to provide an overview of mental health problems that may cause problems in dentistry as well as how dentistry may be in synergy with psychiatry. We are to provide a brief review of the most prevalent and important oral and dental problem in patients with major mental disorders. We also provide a brief note on mental health of dentists.

\section{MENTAL DISORDERS, THEIR TREATMENT AND DENTAL HEALTH}

Dental health and mental health are closely related. Dental/oral health is an important path of general health which contributes to quality of life, verbal communication, self-perception, self-esteem and personal value and social capital. Mouth is directly or symbolically linked to major human drives and passion while the oral mucosa is highly responsive to psychological influences (Pandya \& Nagarajappa 2016). Good teeth are considered as a social and communication asset, supporting verbal expression, social confidence, self-esteem and ability to smile without concern. Oral/dental disorders may 
Table 1. The common dental manifestations in patients with major mental disorders (Tomar et al. 2011, Kisely 2016, Torales et al. 2017)

- eating disorders: erosion of tooth enamel, caries, bleeding gums, angular cheilosis, red sore tongue (anorexia nervosa); anterior tooth erosion, abrasion of teeth and gingivae (bulimia nervosa)

- anxiety disorders: dental cavities, periapical, gingival, periodontal and pulp lesions; cellulitis or abscesses of the oral cavity (commonly due to heavy smoking and poor oral hygiene)

- recurrent depressive disorder: poor oral hygiene, dental cavities, xerostomia; ill-fitting or nonfunctional dental prostheses; various oral-facial pain syndromes

- bipolar disorder: dental cavities, xerostomia, bruxism

- schizophrenia (high risk group): severe forms of dental cavities and advanced generalized periodontal disease (maybe due to subclinical atherosclerosis and heavy smoking); rampant dental decay; multiple missing teeth

- dementia: orofacial trauma usually due to falls, reduction of the flow of saliva, xerostomia; multiple missing teeth; ill-fitting or nonfunctional dental prostheses

- alcoholism: xerostomia, orofacial trauma usually due to falls; candidiasis due to poor nutrition; higher incidence of oral cancer due to heavy smoking; dental attrition/bruxism

affect both body and mind as well as mental disorders may affects oral and body health. Poor dental health may contribute to halitosis, shame, low self-esteem, stigma, discrimination, communication difficulties and social isolation, (Tredget \& Sze 2019). The bacteria related to gingivitis and periodontitis are supposed to be able to pass through the blood stream to the brain and induce memory problems, neurodegenerative disorders and dementia.

On the flip side, mental health has significant ramifications for oral health in many ways. Mental disorders are very prevalent in the population and oral/dental diseases are very prevalent among the psychiatric patients for many reasons. Sadly to say, dental health have a low priority in the context of psychiatric disorders, although the impact of mental disorders and their treatment on oral/dental health may be very detrimental and vice versa dental diseases may have bad effect on course and treatment outcome of mental disorders. Patients with severe mental disorders are 2.5-3 times more likely to have lost all their teeth in comparison to general population (Table 1).

Comorbid dental diseases in psychiatric patients may be related to different risk factors like heavy smoking, abusing alcohol and drugs, a carbohydrate reach diet, lack of self-care and bad oral hygiene, avoidant behavior, negative impact of psychiatric drugs, a negative attitude to dental medicine and fear of dental treatments (Torales et al. 2017, Sagud et al. 2018). Some lifestyle factors such as lack of interest in self-care, poor oral/dental hygiene, cigarette smoking, abuse of alcohol and recreational drugs, acidic drinks and an unhealthy diet rich in sugar and carbohydrates may contribute badly to both oral/dental and mental health (Table 2).

Many mental disorders are commonly associated with oral symptoms such as facial and oral pain and/or preoccupations with dentures. Oral complications of eating disorders such as anorexia nervosa and bulimia nervosa are great challenge to the dentists because of their unique psychodynamic, psychopathological, medical, dental and nutritional characteristics and patterns (Bathia et al. 2015). The early signs of eating disorders are commonly first recognized by dentists as tooth enamel damage induced by purging.

\section{Mental health medicines and dental health}

Treatment with psychiatric drugs are accompanied by adverse events which may produce directly or indirectly oral symptoms and dental damages. The most frequent adverse effect of mental health medication (antidepressants and antipsychotics) is a reduction in Salivary secretions (xerostomia) which is usually, but not always, subjectively experienced as a dry mouth (Denis et al. 2018, Torales et al. 2017, Heaton et al. 2016, Friedlander \& Mahler 2001). Xerostomia increases the risk of dental caries, gum disease and periodontal damages and oral infections like candidiasis, stomatitis, glossitis, and in severe cases salivary gland inflammation (parotitis). These pathological states are followed by difficulties with speech, chewing, poor denture tolerance and stability of dentures. The attachment of germs of the oral origin to different organs, particularly when the immune system is fragile and deficient, can result in serious consequences such as a brain abscess or infective endocarditis or thyroiditis, which is probably underestimated in psychiatric patients (see Denis et al. 2018). Here it is interesting mention dr. Henry Cotton and his stuff who unshakable believed that mental disorders were consequences of untreated infections in body, including dental infections also. They practiced experimental surgical bacteriology on psychiatric patients, including the routine removal of some or all patients' teeth (see Scull 2005).

There are also neurological adverse effects of psychiatric drugs which prevent effective teeth brushing, alter chewing and swallowing, and can induce aspiration problems. Some studies have found an association between type of antipsychotic medication administered and oral hygiene. First generation antipsychotics are more 
Table 2. Mental disorders (ICD-10) commonly encountered in dental clinics (Bathia et al. 2015 - modified)

- Mental and behavioral disorders due to psychoactive substance abuse: Alcohol abuse (F10), Cannabinoids abuse (F12), Heavy smoking(F17), etc.

- Mood (affective) disorders: Bipolar affective disorder (F31); Recurrent depressive disorder (F33), Dysthimia (F34.1)

- Anxiety disorders: Specific phobia (F40.2), Panic disorder (F41.0), Generalized anxiety disorder (F41.1), Obsessivecompulsive disorder (F42), Post-traumatic stress disorders (F43.1)

- Conversion disorder (F44)

- Body dysmorphic disorder; Somatoform disorders: Somatization disorder (F45.0), Hypochondiacal disorder (F45.2), Persistent somatoform pain disorder (F45.4)

- Eating disorders: Anorexia nervosa (F50.0), Bulimia nervosa (F50.2)

likely to cause extrapyramidal symptoms (such as tremor and dyskinesia) which affect the process of tooth brushing (Grinshpoon et al. 2015). Oral dystonias, drooling and bruxism may be the extrapyramidal side effects of a number of drugs used to treat psychiatric illness, primarily the older antipsychotics such as haloperidol and fluphenazine and most slow-release 'depot' medications. These, and the drugs commonly used to counter such side effects, also have anticholinergic properties and may cause a dry mouth (Greenwood \& Meechan 2019). drugs categorized as selective serotonin reuptake inhibitors (SSRIs) may escalate bruxism, while longterm usage of heterocyclic compounds may increase tooth decay due to an increased craving for sweets (Friedlander et al. 2003). Therefore, it is important to provide education about oral health to these patients, as well as using artificial saliva substitutes and stimulants if necessary, topical fluoride, a good oral hygiene, and regular visits to the dentist.

Metabolic adverse effects of some new generation antipsychotics may also be linked with poor oral health. Relationship between metabolic syndrome and oral deficiencies has been recognized during the last decade. Certain infectious oral diseases like periodontal disease seem to be associated with each of the components of the metabolic syndrome (Denis et al. 2018). Diabetes is a risk factor that may contribute to the development of periodontal disease and control of the periodontal infection may contribute to better control of diabetes.

\section{Dental/oral disorders as indicators of decreased mental health}

In recent years, there is an increasing trend in conceptualizing and examining human teeth as possible indicators or biomarkers of decreased mental health. Indeed, Davis et al. (2019) have summarize empirical work across dentistry, anthropology, and archaeology on human tooth development and discussed how teeth preserve a time-resolved record of human life experiences. Specifically, they have articulated how teeth have been examined in these fields as biological fossils in which the history of an individual's early-life experiences is permanently imprinted; this area of research is related to, but distinct from, studies of oral health. They have integrated these insights with knowledge about the role of psychosocial adversity in shaping psychopathology risk to present a working conceptual model, which proposes that teeth may be an understudied yet suggestive new tool to identify individuals at risk for mental health problems following early-life psychosocial stress exposure. There is a research agenda and discussion of future directions for rigorously testing this possibility and with a call to action for interdisciplinary research to meet the urgent need for new biomarkers of adversity and psychiatric outcomes (Davis et al. 2019).

\section{THE NEED FOR COLLABORATION AND OVERCOMING THE PROFESSIONAL GAP BETWEEN PSYCHIATRY AND DENTISTRY}

Access to healthcare is a basic human right, but stigmatization, discrimination and the nature of mental disorders make access to oral/dental health care more difficult. The improving dental health of patients with mental disorders is strongly associated with an improving the quality of life, physical health, social integration in the community and better general treatment outcome in psychiatry. However, psychiatrists fail to address oral health and dental hygiene among their patients due to time constraints, and lack of training and proper skills while dentists in addition to lack of skills for treating special need population show communication barriers and avoidant behaviors. Both psychiatrists and dentists might benefit from person-centered tailored intervention to treat dental health problems among psychiatric patients and mental health problems in dentistry patients.

There is a critical importance of addressing the poor oral health status of people living with mental illness as a means of potentially improving their sense of wellbeing and mental and physical health (Kisely 2016). Yet there is still limited research examining the associations between oral health-related quality of life and having a mental illness. Greater consideration is warranted as to how enhancing oral health in the course of mental healthcare might reduce the burden of a person's ill health (Persson et al. 2009). The role of mental health professionals is important in this regard, yet limited research is available that examines how providers can assist with and promote oral health care for people with 
mental health disorders (Gurbuz et al. 2010, McKibbin et al. 2015). From the point of view of prevention, there should be programs of dental care especially targeted toward this population. These patients and their families should receive education about the importance of oral and dental care and about a correct communication with the treating physician. Some authors believe that an oral examination of mentally ill patients should be considered mandatory (Pitulaj et al. 2019). Furthermore, dental visits are fundamental in providing a holistic attention to this vulnerable group of patients (D'Mello 2003). Seeing that oral health is a very important part of wellbeing in patients with mental illnesses, we should focus on providing these patients an integrated and multidisciplinary attention that includes general practitioners, psychiatrists, dentists, psychologists and nutrition professionals. It has been shown that mental health professionals exhibit ambivalence, reluctance and lack of training in raising oral health issues, despite its acknowledged importance, so more adequate training is in order to overcome this treatment obstacle (Scrine et al. 2018).

\section{Oral/dental health promotion programs in psychiatry}

Poor oral health of psychiatric patients is a major public health issue. Major mental disorders decrease the capacity to plan and execute healthy life styles which involve also dental hygiene and protection. Psychiatric patients often get an evaluation of their basic vitals, somatic and neurologic status, but their oral/dental status is not routinely explored. The severely ill psychiatric patients do not spontaneously ask and get dental treatment and usually do not care properly about their dental status. They simply do not perceive the real meaning of their dental health problems and hence, also due to lack of motivation, do not make the appropriate steps to resolve them. Other than these factors one of the most important factors leading to poor oral hygiene is the recognition of psychiatric disorder itself known as mental health literacy level (MHL) (Guo et al. 2014). MHL level is also known as knowledge or belief of having a mental disorder. This level indicates that the individual is aware of psychiatric illness he or she is suffering from (Wei et al. 2015). Delay in the recognition of MHL leads to poor overall management and prevention ( $\mathrm{Yu}$ et al. 2015). It is interesting that oral health prevention and promotion programs, based on concepts and experience in the general population and transferred on patients with major mental disorders, were no effective enough (Denis et al. 2018). However, specialized oral health promotion programs in psychiatry may for sure contribute significantly to better general treatment results and higher life quality of patients. These programs should include 1. care-giver training; 2 . early screening, 3 . better access to dental care; 4 . therapeutic education of patients and their families; 5 . multidisciplinary treatment; 6. patients and their family participatory (partnership) concept; 7. scientific research and evaluation of (see Denis et al. 2018). The dentist visit is essential to provide a holistic treatment of major mental disorders. It is important to educate psychiatric patients how to use and motivate them to use artificial saliva, mouthwash and topical fluoride, and the treatment of the oral candidiasis when present (Torales et al. 2017). Patient participatory medicine is gradually growing a health care approach. Family physician and dentist awareness of the specific oral/dental health issues in patients with major mental disorders is very important for improving their access the general healthcare systems (Denis et al. 2018). It is useful to have in mind that new development in dental medicine go hand in hand with new approaches in public relations and business, and it is not always easy to know what is the scientific true (see Tavčar \& Dernovšek 2008).

\section{CONSULTATION-LIAISON PSYCHIATRY IN DENTISTRY}

\begin{abstract}
Enhancing oral/dental health for better mental health (Scrine et al. 2017), and vice versa enhancing mental health for better dental health
\end{abstract}

The link between poor dental health and ill mental health is commonly a vicious circle in which persistent pain and inflammation in mouth is leading to anxiety, depression and poor social communication. Given the fact that mental disorders are common problems in general population, it is quite reasonable that dentists frequently meet patients with manifest mental disorders as well as those with disorders that are not obvious or yet identified. These situations can be a major challenge for both patients and dentists. According to some estimation significant psychopathology can be observed in up to $30 \%$ of patients visiting dental clinics (Tomar et al. 2011). However, dentists usually do not ask questions about patients' mental health so psychopathological states in dental patients are usually unrecognized and hence untreated. Oral symptoms may be the first or only manifestation of a mental health problem, such as preoccupation with dentures, burning mouth syndrome, facial pain, etc. A third of patients with temporomandibular joint dysfunction have some of mental disorders. Many psychiatric drugs (antidepressants, antipsychotics) induce adverse events which may produce oral/dental symptoms. Early recognition and appropriate treatment such distressful states would benefit both the individual patients and the health service (see also Tomar et al. 2011).

\section{Dental anxiety and dental phobia}

Oral therapeutic procedures are usually perceived and experienced as being very stressful and inducing acute anxiety, apprehension, tension, irritability and fear (Settineri et al. 2013). Dental anxiety and phobia are related to anticipated harm and avoidance of treatment despite of the lack of correlation between real and per- 
ceived danger (Settineri et al. 2013). These commonly encountered problems in dental practice frequently result in avoidance of dental care and bad oral/dental health. Dental anxiety is dimensional phenomenon that goes from normal to pathological, from tension that a patient cope with to totally avoidance behavior (Halonen et al. 2018). It has been claimed to be the fifth most common cause of anxiety and treating such kind of patients may be a considerable source of stress for the dentist (see Appukuttan 2016). According to some comorbidity studies dental anxiety seems to be closer to mood disorders on the continuum of anxiety disorders and mood disorders (Settineri et al. 2013). It is very important to promptly identify dental anxiety/phobia in patients and treat them properly from the first meeting. Dental anxiety/phobia may result from the previous negative or traumatic experience (classical conditioning), vicarious learning from anxious peers or family members, social learning and frightening portrayals of dentistry procedures in media, vulnerable position of laying back in a dental chair, the personality characteristics of patients, etc (Appukutten 2016).

\section{Atypical odontalgia or Phantom tooth pain}

Atypical odontalgia (AO) or idiopathic odontalgia, a subtype of atypical facial pain or persistent idiopathic facial pain (PIPF), known also as "phantom toothache" is a manifestation of hypersensitivity and tooth pain of unknown cause in radiographically normal teeth (Miura et al. 2018). It is interesting that dental procedures commonly worsen rather than eliminate symptoms while high prevalence of psychiatric comorbidities (about $50 \%$ ) contributes to diagnostic confusion (Miura et al. 2018). Only few patients have severe mental disorders like schizophrenia and bipolar disorder, and dental/ facial pain in patients with somatoform disorders is usually depicted as atypical facial/dental pain. Dental procedures, endodontic treatments, root canal treatments and tooth extraction, may result with later persistent neuropathic facial pain. According to some opinion denture pain represents in some cases the intraoral equivalent of limb stump pain. Atypical odontalgia may be a form of masked depression, known else as depression without depression (for an example see Roš 2003).

\section{Phantom bite syndrome or Occlusal dysesthesia}

Phantom bite syndrome (PBS) is a chronic syndrome characterized by an inability to adapt to changes in dental occlusion although no abnormality is clinically detectable. It is commonly depicted as psychiatric disorder related to delusional disorder, monosymptomatic hypochondrial psychosis, personality disorder, or somatoform disorder (Marbach 1978, Toyufoku \& Kikuta 2006, Watanabe \& Toyofuku 2015). However, PBS is generally not associated with severe mental disorders and absence of a dental trigger predicts a psychiatric comorbidity (Watanabe \& Toyofuku 2015). According to the report of Watanabe and Toyofuku
(2015) only $24(18.5 \%)$ of $63(48.8 \%)$ patients with psychiatric comorbidities had schizophrenia, major depressive disorder, or bipolar disorder. Some authors have suggested proprioceptive dysfunction, false peripheral feed-back and phantom occlusal sensation in the central nervous system. PBS is typically associated with the construction of extensive prosthesis in all age groups and the beginning of orthodontic treatment in adolescents, but it can occur at any stage of dental treatment (Marbach 1996). Despite repeated failures of dental surgery, patients persist in seeking help and bite correction from a succession of dentists. Good news is that serotonin and norepinephrine reuptake inhibitors like milnacipran may significantly help (Toyufoku \& Kikuta 2006). PBS with left-sided occlusal discomfort seems to be associated with a patients' history of depression (Shinohara et al. 2020).

\section{Burning mouth syndrome}

Burning mouth syndrome (BMS) is a condition manifested as a burning pain or hot sensation in the tongue or lips or gums or more widespread in mouth. Patients commonly describe the burn as feeling like a hot drink scald. Etiology is related to the way the tongue transmits warmth, cold and taste to the brain. The primary BMS is commonly associated with depressive and anxiety disorders (Bathla et al. 2015, Sikora et al. 2018). During clinical evaluation of BMS somatization should be taken into consideration. The secondary BMS may be associated with allergies to some foods or dental products, consuming huge amount of acidic foods or drinks, dry mouth, diabetes mellitus, hypothyroidism, gastroesophageal reflux disease, mouth infections, nutritional deficiencies, overusing mouthwash or abrasive oral hygiene products. Low dose antidepressants may be helpful.

\section{Halitosis, halitophobia and delusional bad odor}

Halitosis (Latin halitus - breath; Greek suffix osis pathological process), fetor ex ore, bad breath or oral malodor is an oral/dental health condition which may negatively influence on social contacts and might induce mental problems and psychological complications. Usually, halitosis is differentiated in genuine halitosis and delusional halitosis, while genuine halitosis may be physiological (morning) halitosis and pathological halitosis. Physiological halitosis is related to the food remnants, exfoliated epithelial cells and saliva stagnation which lead to bacterial accumulation on the dorsal surface of the tongue that is seen as the tongue coating (Heboyan et al. 2019). Etiology of pathological halitosis may be related to both, systemic and oral conditions, but in general about $85 \%$ cases are related to an oral cause. Oral causes involve poor oral health care, dry mouth, heavy smoking, deep carious lesions, periodontal diseases, oral infections, peri-implant diseases, pericoronitis, mucosal ulcerations, impacted food or debris, and tongue coating (Corteli et al. 2008). 
Pathologic subjective halitosis or pseudo-halitosis represents a halitosis complaint without objective confirmation of bad odor by others or by halitometer measurement, and it is usually associated with social anxiety disorder or obsessive-compulsive disorder (Tsuruta et al. 2017). Halitophobia (the fear of having bad breath) is a condition manifested as an excessive preoccupation with the belief of having halitosis. It may be related to various psychiatric disorders such as delusional disorder, hypochondria, obsessive compulsive disorder and olfactory reference syndrome, a sub-category of body dysmorphic disorder.

\section{Innovation in dental clinics and psychodentology}

Dental patients commonly suffer from both physical diseases and mental disorders and there is a need for closer collaboration between dentists and psychiatrists (Ray et al. 2015). Consultation-Liasion (CL) psychiatry is fast developing and well-recognized branch of psychiatry and psychosomatic medicine that covers the interface between physical and mental health promoting holistic, integrative and personalized approach. Comorbidity of dental diseases and mental disorders influences significantly on treatment outcome both in psychiatry and dentistry. The dentist's ability to support better quality of life and self-esteem of psychiatric patients can be a very rewarding experience in dentistry-psychiatry cooperation (Elhadad 2017). Psychiatric-dental consultation-liaison (PDCL) services could be of great help to both psychiatric and dental patients as well as to their physicians. PDLC could provide a framework for better understanding of mental disorders by dentists, early diagnosis and more successful treatment patients with comorbidities and better outcome as well as better understanding of dental health by psychiatrists and prevention of dental comorbidities in psychiatric patients. PDLC services could offer behavioral relaxation techniques, hypnosis, yoga or meditation, biofeedback, rational-emotional-behavioral therapy (REBT), cognitive behavioral therapy (CBT), and pharmacotherapy with anxiolitics, antidepressants, hypnotics and antipsychotics (Pandya \& Nagarajappa 2016).

Mental health of dentists. In some circumstance dentistry may be a very stressful profession and dentists commonly perceive dentistry as being more stressful than other occupations (Rada \& Johnson-Leong 2004). Prevention of burnout syndrome, alcoholism and substance abuse, anxiety and depressive disorders requires that dentins learn coping strategies in time which include business management, stress management and communication skills.

\section{CONCLUSIONS}

Comorbidity of somatic diseases and mental disorders has been the subject of growing interest, but without or with little interest for the issue of dental/oral health. Dental comorbidity in psychiatric patients may be related to the changes in the saliva quality and quantity, changes in oral and gastrointestinal microbiota, immune and endocrine dysfunctions and psychomotor problems. Medical professionals should be aware of the impact of mental disorders and their treatment on oral health as well as of the impact of oral/dental disorders on mental health and treatment outcome in psychiatry. Management of dental anxiety/phobia, atypical odontalgia, occlusal dysesthesia halitosis, halitophobia should be an interdisciplinary procedure among dentists, psychiatrists, psychologists and general/ family physicians. Regular dental checks and a proper oral hygiene is vital for well-being and good mental health. Dental health and quality of dental health care should be a basic human right rather than a privilege. Modern dentistry may significantly contribute to better treatment outcome in psychiatry and higher quality of life of psychiatric patients. Better coordination between medical, dental and psychiatric services is needed for better general health of population.

\section{Acknowledgements: None.}

\section{Conflict of interest: None to declare.}

\section{Contribution of individual authors:}

Zdenko Šarac: initiative, concept and design of the article, writing manuscript, particularly the part dedicated do dental aspects, approval of the final version.

Ružica Zovko \& Marina Čurlin: literature searches, comment on concept and design of article, approval of the final version.

Pavo Filaković: concept and design of the article, writing manuscript, particularly the part dedicated to psychiatric aspects, approval of the final version.

\section{References}

1. Appukutten D: Strategies to manage patients with dental anxiety and dental phobia: literature review. Dovepress 2016; 8:35-50 doi https://doi.org/10.2147/CCIDE.S63626

2. Arigbede AO, Babatope BO \& Bemidele MK: Periodontitis and systemic diseases. A literature review. Journal of Indian Society of Periodontology 2012; 16:487-491. doi:10.4103/0972-124X106878

3. Bathia M, Chandna S, Mehta DS \& Grover HS: Dentistry and Psychiatry: It's time to bridge the gap. Delhi Psychiatry Journal 2015; 18:1-5

4. Corteli JR, Barbosa MDS \& Westphal MA: Halitosis: A review of associated factors and therapeutic approach. Braz oral Res 2008; 22(Spec Iss 1):44-54

5. Danis F, Pelletier JF, Chauvet-Geliner JC, Rude N\& Trojak B: Oral health is a challenging problem for patients with schizophrenia: A narrative review. Iran J Psychiatry Behav Sci 2018. e8062 doi:10.5812/ijpbs. 8062 
6. Davis KA, Mountain RV, Pickett OR, Den Besten PK, Bidlack FB, Dunn EC: Teeth as Potential New Tools to Measure Early-Life Adversity and Subsequent Mental Health Risk: An Interdisciplinary Review and Conceptual Model. Biol Psychiatry 2019. pii: S0006-3223(19)31780-9

7. D'Mello DA: Are your patients depressed? Implications for dental practice. J Mich Dent Assoc 2003; 85:26-32

8. Elhadad AA: A study of dental diseases in psychiatric patients: is there a relationship? Egyptian Journal of Psychiatry 2017; 38:143-146

9. Feinmann $C \&$ Harrison S: Liaison psychiatry and psychology in dentistry. Journal of Psychosomatic Research 1997; 43:467-476

10. Friedlander AH, Mahler ME: Major depressive disorder: Psychopathology, medical management and dental implications. J Am Dent Assoc 2001; 132:629-638

11. Friedlander AH, Friedlander IK, Gallas M, Velasco E: Late-life depression: Its oral health significance. Int Dent J 2003; 53:41-50

12. Grown S, Greenwood $M$ \& Meechan JG: General medicine and surgery for dental practitioners. Part 5 psychiatry. British Dental Journal 2010; 209:11-16

13. Glass M: The need for collaboration between psychiatry and dentistry. The American Journal of Psychiatry, published online 2018. https://doi.org/10.1176/appi.ajp-rj.2018.130604

14. Grinshpoon A, Zusman SP, Weizman A \& Ponizovsky AM: Dental health and the type of antipsychotic treatment in patients with schizophrenia. Isr J Psychiatry Related Sci 2015; 52:114-118

15. Guo Y, Logan HL, Dodd VJ, Muller KE, Marks JG, Riley III JL: Health literacy: a pathway to better oral health. American Journal of Public Health 2014; 104:e85-e91

16. Halonen H, Nissinen J, Lehtiniemi H, Salo T, Riipinen P \& Miettunen J: The associationen between dental anxiety and psychiatric disorders and symptoms: A systematic review. Clinical Practice \& Epidemiology in Mental Health 2018; 14:207-222. doi: $10.2174 / 1745017901814010207$

17. Heaton LJ, Swigart K, McNelis G, Milgrom P, Downing DF: Oral health in patients taking psychotropic medications: Results from a pharmacy-based pilot study. $J$ Am Pharm Assoc 2016; 56:412-417.e1

18. Heboyan A, Avetisyan A \& Vardanyan A: Halitosis as an issue of social and psychological significance. Journal of research in Medical and Dental Science 2019; 7:33-40

19. Horst G: Dental care in psychiatric hospitals in the Netherlands. Spec Care Dentist 1992; 12:63-66

20. Jakovljevic $M$ \& Borovecki F: Epigenetics, resilience, comorbidity and treatment outcome. Psychiatr Danub 2018; 30:242-253. https://doi.org/10.24869/psyd.2018.242

21. Kisely S: No mental health without oral health. The Canadian Journal of Psychiatry 2016; 61:277-282

22. Löffler W, Kilian R, Toumi M, Angermeyer MC: Schizophrenic patients' subjective reasons for compliance and noncompliance with neuroleptic treatment. Pharmacopsychiatry 2003; 36:10512

23. Marbach JJ: Phantom bite syndrome. Am J Psychiatry 1978; 135:476-479

24. Marbach JJ: Orofacial phantom pain: theory and phenomenology. J Am Dent Assoc 1996; 127:221-229

25. Matevosyan NR: Oral health of adults with serious mental illnesses: a review. Community Ment Health J 2010; 46:553-62
26. McKibbin C, Kitchen-Andren K, Lee A, Wykes $T$ \& Bourassa K: Oral health in adults with serious mental illness: Needs for and perspectives on care. Community Mental Health Journal 2015; 51:222-228

27. Miura A, Tu TTH, Shinohara Y, Mikuzuki L, Kawasaki K, Sugawara S, Suga T, Watanabe T, Watanabe M, Umezaki Y, Yoshikawa T, Motomura H, Takenoshita M, Maeda H \& Toyofuku A: Psychiatric comorbidities in patients with atypical odontalgia. Journal of Psychosomatic Research 2018; 104:35-40

28. Muzycka BC, Glass $M$ \& Glass OM: Oral health in electroconvulsive therapy: a neglected topic. I ECT 2017; 33:12-15 Crossref, Google Scholar

29. Pandya $D$ \& Nagarajappa AK: A rare case report on atypical odontalgia with psychological implications. Ind $J$ Med Res Rev 2016; 4:261-263

30. Persson K, Axtelius B, Söderfeldt B, Östman M: Monitoring oral health and dental attendance in an outpatient psychiatric population. Journal of Psychiatric and Mental Health Nursing 2009; 16:263-71

31. Pitułaj A, Kiejna A, Dominiak M: Negative synergy of mental disorders and oral diseases versus general health. Dent Med Probl 2019; 56:197-201

32. Rada RE \& Johnson-Leong C: Stress, burnout, anxiety and depression among dentists. JADA 2004; 135:788-794

33. Ray PK, Ray (Bhattacharya) S, Makhal M, Majumder U, De $S \&$ Ghosh S: Prevalence of psychiatric co-morbidity among patients attending dental OPD and the role of consultation-liaison psychiatry in dental practice in a tertiary care general hospital. Indian $J$ dent (serial online) 2015; 6:32-36 http://www.ijdentistry.com/text.asp?2015/6/1/32/151707

34. Roš LT: Pain mask in masked depression in the form of selective toothache - Own experience: Case report. Psychiatr Danub 2003; 15:29-31

35. Sagud M, Vuksan Cusa B, Jaksic N, Mihaljevic Peles A, Rojnic Kuzman M, Pivac N: Smoking in Schizophrenia: an Updated Review. Psychiatr Danub 2018; 30(suppl 4):216-223

36. Scrine C, Durey A, Slack-Smith L: Enhancing oral health for better mental health: Exploring the views of mental health professionals. International journal of mental health nursing. 2018; 27:178-86

37. Scull A: Madhouse - A Tragic tale of Megalomania and Modern Medicine, Yale University Press, New Haven, USA, 2005

38. Settineri S, Mallamace D, Muscatello MRA \& Zoccali R: Dental anxiety, psychiatry and dental treatment. Open Journal of Psychiatry 2013; 3:168-172

39. Shinohara Y, Umezaki Y, Minami I, Watanabe M, Miura A, Mikutsuki L, Kawasaki K, Sugawara S, trang TTH, Suga T, Watanabe T, Yoshikawa T, Takenoshita M, Motomura $H$ \& Toyofuku A: Comorbid depressive disorders and left-side dominant occlusal discomfort in patients with phantom bite syndrome. Journal of Oral Rehabilitation 2020; 47:36-41. doi: 10.1111/joor.12872

40. Sikora M, Verzak Z, Matijevic M, Vcev A, Siber S, Music L \& Carek A: Anxiety and depression scores in patients with burning mouth syndrome. Psychiat Danub 2018; 30:466-470 https://doi.org/10.24869/psyd.2018.466

41. Simunovic Filipcic I, Filipcic I: Schizophrenia and physical comorbidity. Psychiatr Danub 2018; 30(suppl 4):152157

42. Tavčar $R \&$ \& Dernovšek M: Clinicallly tested toothpaste and other fairy tales - A critical approach to reading 
pharmaceutical advertisements and professional papers. Psychiatr Danub 2008; 20:249-250

43. Tomar B, Bhatia NK, Kumar P, Bhatia MS \& Shah RJ: The psychiatric and dental interrelationship. Delhi Psychiatry Journal 2011; 14:138-142

44. Torales J, Barrios I \& Gonzales I: Oral and dental health issues in people mental disorders. Medwave 2017; 17:7045. doi: 10.5867/medwave.2017.08.7045

45. Toyofuku A \& Kikuta T: Treatment of phantom bite syndrome with milnacipran - a case series. Neuropsychiatr Dis Treat 2006; 2:387-390. doi: 10.2147/nedt-2006.2.3.387

46. Tredget $J$ \& Sze TW: Raising awareness of oral health care in patients with schizophrenia. Nursing Times (online) 1019; 115:21-25. www.nursingtimes.net

47. Tsuruta M, Takahashi T, Tokunaga M, Iwasaki M, Kataoka S, Kakuta S, Soh I, Awano S, Hirata H, Kagawa M
\& Ansai T: Relationship between pathologic subjective halitosis, olfactore reference syndrome, and social anxiety in young Japanese women. BMC Psychology 2017; 5:7. doi:10.1186/s40359-017-0176-1

48. Watanabe $M$ \& Toyofuku A: Psychiatric comorbidities and psychopharmacological outcomes of phantom bite syndrome. Journal of Psychosomatic Research 2015; 78:255-259.

https://doi.org/10.1016/j.jpsychores.2014.11.010

49. Wei Y, McGrath PJ, Hayden J, Kutcher S: Mental health literacy measures evaluating knowledge, attitudes and help-seeking: a scoping review. BMC Psychiatry 2015; 15:291

50. Yu Y, Liu Z-w, Hu M, Liu H-m, Yang JP, Zhou L, et al: Mental health help-seeking intentions and preferences of rural Chinese adults. PloS One 2015; 10:e0141889

Correspondence:

Zdenko Šarac, MD

Faculty of Dental Medicine, Department of Head and Neck Diseases,

University of Mostar

Bijeli brijeg bb, 88000 Mostar, Bosnia and Herzegovina

E-mail: z-sarac@hotmail.com 